\title{
Laparoscopic Management of Acute Intestinal Obstruction
}

\section{Tamer M Said Salama*}

Department of Surgery, Ain Shams University, Egypt

\begin{abstract}
Background: With the beginning of laparoscopic era at the end of last century acute intestinal obstruction was one of relative contraindication of laparoscopy; however, with development of minimal invasive surgeries new vision was born for the use of laparoscopy in the management of acute intestinal obstruction.
\end{abstract}

Aim of the study: This study aiming to evaluate the role of laparoscopy in management of acute intestinal obstruction as regard indication, technique, contraindication and complication.

Material and methods: A systematic literature search was carried out using the Medline database and the search terms "laparoscopy" or "laparoscopic approach" and "bowel obstruction." Only adult studies published in English between 1990 and 2016 were included. Studies were excluded if data specific to outcomes for laparoscopic management of acute intestinal obstruction could not be extracted.

Conclusion: Laparoscopy is safe and effective tool for the management of acute intestinal obstruction provided if it performed by skilled surgeons and in selected patients.

Keywords: Laparoscopy; Laparoscopic approach; Bowel obstruction

\section{Introduction}

Acute intestinal obstruction is defined as sudden interruption of forward flow of intestinal contents. This interruption may occur at any point in gastrointestinal tract. Intestinal obstruction represented in $15 \%$ of patients presented with acute abdominal pain to ER [1]. It is a serious health problem, which may lead to intestine perforation and generalized peritonitis/septic shock/severe metabolic disorders that can cause acute dehydration, acute renal failure, cardiac arrhythmias, and acute heart failure with high mortality rate in old age [2].

\section{Classification of intestinal obstruction [3]}

There are multiple classifications for intestinal obstruction based on:

A) Presence of absence of a physical impediment

- Dynamic or mechanical obstruction: There is a physical impediment to the flow of the luminal contents.

- A dynamic (ileus) obstruction: There is no physical impediment; rather there is a failure of normal peristalsis.

B) The location of the cause of the obstruction

- Intraluminal i.e., inside the lumen, for example fecal impaction, meconium plug, meconium ileus, ascaris whorms.

- Intramural i.e., in the wall of the bowel, for example; tumors, intussusception, strictures, anastomotic stenosis and edema.

- Extraluminal i.e., outside the lumen, for example; tumors, adhesions, herniation, gravid uterus, abscess collections.

C) The segment of the bowel involved

- High or small bowel obstruction.

- Low or large bowel obstruction.

D) The degree of obstruction

- Complete: no passage of stool or flatus.

- Partial or subacute: there is still passage of at least flatus or small quantity of stool.
E) The rapidity of evolution of the symptoms.

- Acute: rapidly evolving symptoms, which small is more common with small bowel obstruction?

- Chronic: slowly evolving symptoms, pain may not be prominent, which is more common with large bowel obstruction.

- Acute on top of chronic: this is when the symptoms were slowly progressive but suddenly become accentuated or accelerated. This suggests an obstruction that involved the large bowel but has progressed to involve the small bowel by reflux through an incompetent ileo-caecal valve.

F) The number of points of obstruction

- Simple obstruction: when there is failure of forward movement of intestinal content due to presence of physical impediment closing the lumen, but the luminal content can move backwards.

-Closed loop: it occurs when the bowel lumen is obstructed in two points in which intestinal content can't move forward or backwards. For example as seen in volvulus, herniating intestinal loop in obstructed hernia or in left colonic tumor with a competent ileo-caecal valve.

- Strangulated obstruction: when there is compromise to the blood supply of the obstructed segment.

\section{Etiology}

Bowel obstruction may be functional obstruction, due to bowel wall or splanchnic nerve dysfunction which is common with generalized peritonitis and electrolyte disturbance, or mechanical obstruction due to a mechanical barrier. The most common causes of intestinal

*Corresponding author: Dr Tamer M Said, Department Of Surgery, Faculty of Medicine, Ain Shams University, 16 Foud Badwany Street Nasr City, Cairo, Egypt Tel: 01113623458; E-mail : drtamer1981@hotmail.com

Received February 23, 2017; Accepted March 21, 2017; Published March 28 2017

Citation: Salama TMS (2017) Laparoscopic Management of Acute Intestinal Obstruction. Surgery Curr Res 7: 290. doi: 10.4172/2161-1076.1000290

Copyright: (๑) 2017 Salama TMS. This is an open-access article distributed under the terms of the Creative Commons Attribution License, which permits unrestricted use, distribution, and reproduction in any medium, provided the original author and source are credited. 
obstruction include adhesions, neoplasms, and herniation (Figure 1). Post-operative adhesions are the most common cause of small bowel mechanical obstruction, it represented in 40-50 percent of cases [4].

\section{Pathophysiology}

The main concerns of intestinal obstruction pathophysiology are its effect on whole body fluid/electrolyte balances and the mechanical effect that increased pressure has on intestinal perfusion $[5,6]$.

Fluid loss after vomiting, bowel edema, and loss of absorptive capacity leads to dehydration. Loss of electrolyte especially gastric potassium, hydrogen, and chloride ions, and significant dehydration stimulates renal proximal tubule reabsorption of bicarbonate and loss of chloride, which end by metabolic alkalosis. In addition, intestinal stasis leads to overgrowth of intestinal flora in the small bowel which leads to bacterial translocation across the bowel wall [7].

Ongoing dilation of the intestine increases luminal pressures. When luminal pressures exceed venous pressures, loss of venous drainage causes increasing edema and hyperemia of the bowel. This may eventually lead to compromised arterial flow to the bowel, causing ischemia, necrosis, and perforation. A closed-loop obstruction, in which a section of bowel is obstructed proximally and distally, may undergo this process rapidly, with few presenting symptoms. Intestinal volvulus, the prototypical closed-loop obstruction, causes torsion of arterial inflow and venous drainage, and is a surgical emergency [8].

\section{Clinical Presentation}

The classical clinical features is colicky abdominal pain, nausea and emesis, abdominal distension, and progressive obstipation (Severe constipation caused by intestinal obstruction), in addition to some general symptoms according to the stage of the disease [9].

\section{Pain}

The pain is relatively sudden in onset, sharp, periumbilical and may progressively diminish because of bowel fatigue. Emesis may temporarily ameliorate the pain by deflating distended bowel. The pain becomes more intense and unremitting if intestinal ischemia or perforation occurs. Closed-loop obstruction often presents with pain out of proportion to the abdominal signs because of concurrent mesenteric ischemia [9].

\section{Pie chart showing relative frequency of the underlying}

\section{diagnosis of intestinal obstruction.}

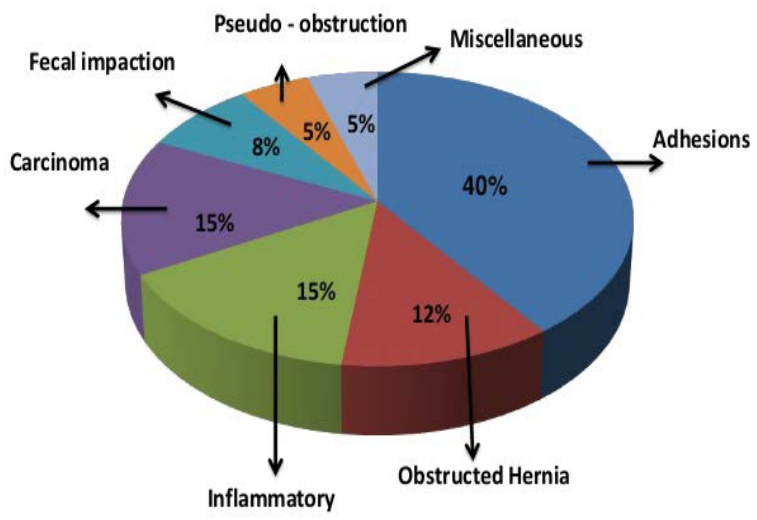

Figure 1: Causes of acute intestinal obstruction [5].

\section{Vomiting}

The distal obstruction has longer interval between the onset of symptoms and the appearance of nausea and vomiting. As obstruction progress, the character of vomitus alters from digested food to feculent material as a result of the presence of enteric bacterial overgrowth [10].

Vomiting is a marked feature of high small-bowel obstruction but is rarely encountered in colonic obstruction. Vomiting, together with the sequestration of fluid in the dilated loops, rapidly leads to dehydration with significant water and electrolyte deficits, particularly $\mathrm{K}^{+}$and $\mathrm{Cl}^{\text {. }}$. The dehydration leads to prerenal azotaemia, with elevation of blood urea and reduced urine output [2].

\section{Constipation}

Symptoms of constipation may not be apparent initially because residual gas and stool in bowel distal to the obstruction may continue to evacuate. In partial obstruction; patient may continue to pass flatus and faeces, in conjunction with the other symptoms [11].

\section{Distention}

The degree of distention depends on the site of the obstruction and upon the time factor. When obstruction occurs in upper small bowel the distention not obvious and is mainly present on the stomach and epigastrium region. In low small bowel obstruction the distention becomes moderate and appears in central abdomen, In large bowel obstruction, the distention becomes generalized all over the abdomen especially the flanks [12].

\section{General symptoms}

Symptoms may range from minimal discomfort with few physical abnormalities to toxicity and sepsis. The development of rigors, high fever, or systemic toxicity suggests that the obstruction may be complicated by intestinal necrosis or perforation [13].

\section{Diagnostic Testing and Imaging}

\section{Laboratory tests}

Laboratory evaluation of patients with suspected obstruction should include a complete blood count and metabolic panel. Hypokalemic, hypochloremic metabolic alkalosis may be noted in patients with severe emesis. Elevated blood urea nitrogen levels are consistent with dehydration, and hemoglobin and hematocrit levels may be increased. The white blood cell count may be elevated if intestinal bacteria translocate into the bloodstream, causing the systemic inflammatory response syndrome or sepsis. The development of metabolic acidosis, especially in a patient with an increasing serum lactate level, may signal bowel ischemia [14].

\section{Radiological testes}

Plain X-rays: Radiography accurately diagnoses intestinal obstruction in approximately $60 \%$ of cases [15], and its positive predictive value approaches $80 \%$ in patients with high-grade intestinal obstruction [16]. However, plain abdominal films can appear normal in early obstruction and in high jejunal or duodenal obstruction. The most specific for small bowel obstruction is the tirade of dilated small bowel loops ( $>3 \mathrm{~cm}$ in diameter), air fluid levels seen on upright films (Figure 2) [17].

Contrast radiography: Contrast studies, such as a small bowel follow-through, can be helpful in the diagnosis of a partial intestinal obstruction in patients with high clinical suspicion and in clinically 
stable patients in whom initial conservative management was not effective [18]. The use of water-soluble contrast material is not only diagnostic, but may also be therapeutic in patients with partial smallbowel obstruction [19].

Abdominal ultrasonography: Ultrasonography remains a valuable investigation for unstable patients with an ambiguous diagnosis and in patients for whom radiation exposure is contraindicated, such as pregnant women [20].

Computed tomography (CT): CT is appropriate for further evaluation of patients with suspected intestinal obstruction in whom clinical examination and radiography do not yield a definitive diagnosis. CT is sensitive for detection of high-grade obstruction (up to $90 \%$ in some series) [21] and has the additional benefit of defining the cause and level of obstruction in most patients (Figure 3) [22].

Magnetic resonance imaging: The imaging signs of (MRI) are similar to CT scanning but MRI is superior in demonstrating gut wall edema and peritoneal fluid. In comparison with the intra operative results MRI showed a sensitivity of $87.5 \%$ and specificity of $92 \%$ [23].

\section{Laparoscopic Management Acute Intestinal Obstruction}

With the beginning of laparoscopic era at the end of last century acute intestinal obstruction was one of relative contraindication of laparoscopy, however with development of minimal invasive surgeries new vision was born for the use of laparoscopy in the management of acute intestinal obstruction [24].

Bastug et al. [25] reported the first laparoscopic lysis of a single adhesive band causing intestinal obstruction (Figure 4). Despite this, the surgical community has been slow to adopt laparoscopy in the treatment of this common disorder.

\section{Limitations}

The main limitations of this procedure are access and visualization of the peritoneal cavity in the setting of small bowel distension. Most of the procedure complications are related to the blind placement of the Veress needle or sharp primary trocar into the abdomen when performing a technique referred to as "closed" laparoscopy [26].

\section{Selection criteria for laparoscopy uses in intestinal obstruction [27]}

a) Patients presented early with IO.
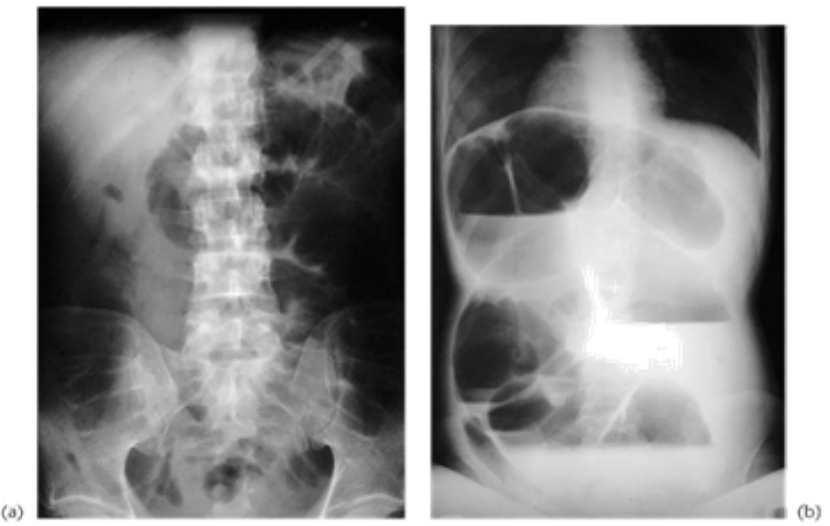

Figure 2: Plain $X$ ray showed huge intestinal dilation, not candidate for laparoscopic management [2].

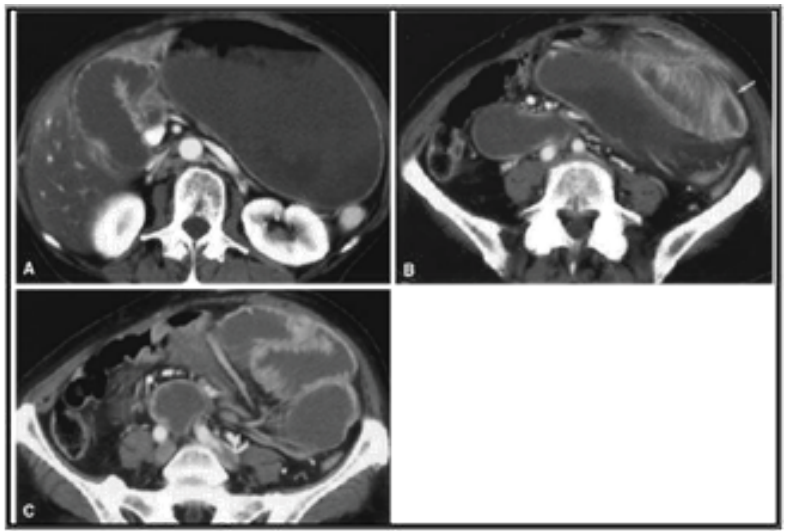

Figure 3: Closed-loop obstruction [41].

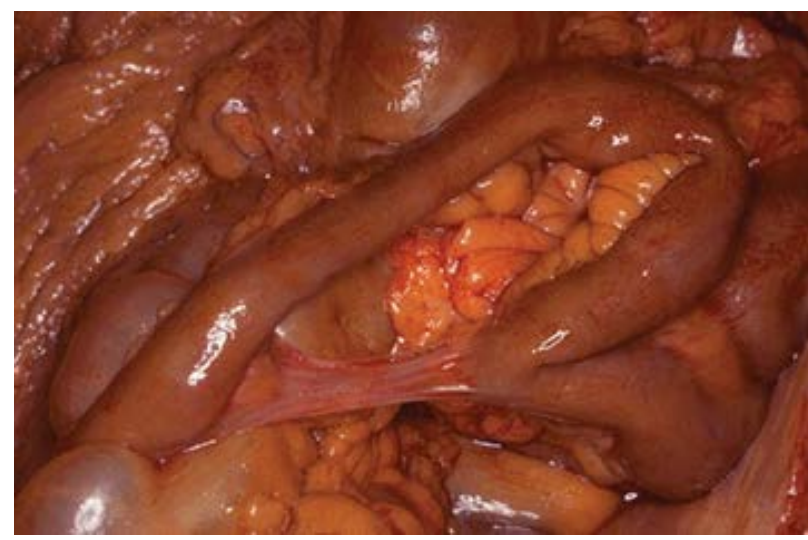

Figure 4: Adhesive intestinal obstruction [42].

b) When proximal intestinal obstruction is suspected.

c) When Partial intestinal obstruction is suspected.

d) When single adhesive band is anticipated.

e) Localized distension on radiography.

f) Patients with good general condition of the patient i.e., no sepsis

g) Patients with small number of previous abdominal surgical procedures.

h) Patients respond well to preoperative preparation.

\section{Contraindications of the procedure [28]}

a) Complicated intestinal obstruction i.e., patient in septic shock, suspecting strangulated bowel loop.

b) Severely distended bowel loop not responding to conservative treatment.

c) When suspecting malignancies.

d) When suspecting bowel ischemia or necrosis, closed-loop obstruction, and perforation.

e) The overall absence of selection criteria.

f) Lack of experience in performing advanced laparoscopy in an emergency setting. 
g) Other absolute contraindications are severe comorbidities, as cardiovascular, respiratory and hemostatic disease.

\section{Preoperative preparation}

Preoperative preparations in the form of adequate fluid and electrolyte replacement, prophylaxis broad spectrum antibiotics, anti-thrombotic prophylaxis, is the corner stone if we want to use laparoscopy in the management of acute intestinal obstruction [29].

Nasogastric decompression is the corner stone in laparoscopic management of intestinal obstruction. As well as facilitating decompression proximal to the obstruction, they also reduce the risk of subsequent aspiration during induction of anesthesia and post extubation. Some authors have advocated nasointestinal "long tube" intubation over nasogastric "short tube" intubation. The rationale behind this theory is that a long tube placed distal to the pylorus promotes more complete intestinal decompression, allowing the now decompressed bowel increased mobility and more opportunities to become unobstructed [30].

A urinary catheter is not needed when the patient presents early and the operation is simple. When the diagnosis has been delayed, a urinary catheter is essential to monitor rehydration before and after the operation. It should be remembered that urine production during the laparoscopy itself is usually diminished. A urinary catheter is also essential in case of postoperative epidural analgesia [31].

\section{Tips and tricks of the procedure}

It's preferred to put the first trocar away from any previous abdominal scar either via open technique (Hasson's technique) or by "optical-access" trocars (visiport). These trocars were designed to decrease the risk of injury to intra-abdominal structures by allowing the surgeon to visualize abdominal wall layers during placement [32].

a) The pneumoperitoneum should never exceed $12 \mathrm{mmHg}$.

b) Free fluid should be aspirated and sent for gram staining, amylase, bilirubin, and culture.

c) To facilitate exposure, table tilt, and external manual compression of the abdominal wall should be used.

d) Bowel should be examined for perforation and signs of ischemia.

e) Exposure can be achieved by pushing with closed instruments rather than by grasping with the instruments. The small bowel should be inspected in a retrograde fashion commencing at the cecum and decompressed bowel until the point of transition is identified. Cold scissors should be used to divide matted adhesions or a band. Energybased devices should be avoided to divide adhesions [24].

f) After initial diagnostic laparoscopy, additional working ports (usually two 5-mm ports) can be placed under direct vision. The entire abdominal cavity should be inspected carefully, with attention directed towards the collapsed distal bowel to identify the cause of obstruction [24].

g) Care should be taken to avoid handling the distended bowel where possible, and traumatic bowel graspers should be used to minimize the risk of bowel injury. The view can be enhanced by tilting the patient in several directions during surgery to maximize exposure. Once the cause of the obstruction identified examined from the caecum to the ligament of Treitz to ensure that the obstruction did not involve multiple levels of the small bowel. h) If intestinal injury occurs or the surgeon notices ischemic or necrotic bowel conversion to open laparotomy is necessary.

\section{Clinical parameters which predict success of laparoscopic intervention [33]}

a) Proper preoperative preparation of the patients.

b) Temporarily improve/resolve after placement of a nasogastric tube but recur or prevent the patient from tolerating an oral diet.

c) Previous operative note did not describe massive or severely problematic adhesions.

d) Ability to gain safe access to the peritoneal cavity.

e) Obstructed segments not fixed to the retroperitoneum.

f) Early laparoscopic management within 24 hours from the onset of symptom.

\section{Advantages of the procedure [34]}

a) Shorter hospital stay.

b) Shorter recovery time.

c) Reduce postoperative morbidities i.e., postoperative pain from the incisions, wound infection, incisional hernia.

d) Faster return to normal diet.

e) Faster return to work or normal activity.

f) Better cosmetic healing

g) Furthermore it is approved to be a diagnostic tool of difficult pathology e.g. internal hernia, venous thrombosis and other rarities.

h) There are clinical data backed up by several animal studies supporting the hypothesis that laparoscopy leads to a decreased rate of adhesion formation as compared to laparotomy.

\section{Compilations of the procedure}

a) Iatrogenic intestinal injury one of the most dreaded complications in laparoscopic management of SBO is intestinal injury. In a single comparative study, the risk of perforation was $27 \%$ in the laparoscopic group which was clearly higher than in the open group [28].

b) Strickland et al. [35] found that duration of surgery longer than 120 minutes; intraoperative perforation, bowel necrosis, and conversion to laparotomy were significant predictors of postoperative morbidity.

\section{Review of Articles}

i) In study done by Farinella et al. the performed a review without any language restrictions considering international literature indexed from 1980 to 2007 in Medline. The literature searches were carried out using the following keywords: laparoscopic adhesiolysis, laparoscopic adhesiolysis, laparoscopic management and small bowel obstruction, they conclude that Laparoscopic adhesiolysis in small bowel obstruction is feasible but can be convenient only if performed by skilled surgeons in selected patients [36].

ii) In prospectively study done by Henry et al. 61 patients presented with acute small bowel obstruction. Laparoscopic techniques (LAP) alone were successfully used to complete 41 cases (67\%). Twenty patients $(33 \%)$ were converted to either minilaparotomy (7 patients (35\%)) or standard midline laparotomy (13 patients (65\%)) [37]. 
iii) In study done by Franklin et al. 167 patients underwent laparoscopy for diagnosis and/or treatment of intestinal obstruction. Laparoscopy successfully diagnosed the site of obstruction in all patients. In addition, 154 patients (92.2\%) were successfully treated laparoscopically without conversion to laparotomy [38].

iv) In study done by Donal, A systematic literature search was carried out using the Medline database and the search terms laparoscopy or laparoscopic approach and bowel obstruction. A laparoscopic approach was attempted in 2,005 patients with acute IO, they conclude that laparoscopy is a feasible and effective treatment for acute SBO with acceptable morbidity. Further studies are required to determine its impact on recurrent IO [39].

v) In a retrospective study done by Jennifer et al., reported our experience of laparoscopic management of acute small bowel obstruction was undertaken Laparoscopic treatment was successful in $78 \%$ of patients including one laparoscopy-assisted procedure [24].

vi) A retrospective review was performed of all patients with acute SBO in whom laparoscopic treatment was attempted, Conclusions: Laparoscopy is a safe and effective procedure for the treatment of acute SBO in selected patients [35].

vii) In study done by Bashar et al., 1061 cases suffering from intestinal obstruction underwent laparoscopic exploration. They concluded that Laparoscopy is an effective procedure for the treatment of acute small bowel obstruction with acceptable risk of morbidity and early recurrence [40].

\section{Conclusion}

Laparoscopy is safe and effective tool for the management of acute intestinal obstruction provided if it performed by skilled surgeons and in selected patients.

\section{References}

1. Patrick GJ, Manish R (2011) Evaluation and Management of Intestinal Obstruction. Am Fam Physician 83: 159-165.

2. Cuschieria (2003) Clinical features of intestinal obstruction, acute abdomen, clinical surgery (2nd edn). Blackwell, USA. pp: 166-180.

3. Kulaylat MN, Doerr RJ (2007) Small bowel obstruction. Gastrointest Endosc Clin N Am 2: 323-329.

4. Shelton BK (1999) Intestinal obstruction. AACN Clin Issues 10: 478-491.

5. http://www.slideshare.net/chirantanist26/introduction-of-intestinal-obstructionpallavi-shekhar-medical-college-kolkata

6. Wright HK, O'Brien JJ, Tilson (1971) Water absorption in experimental closed segment obstruction of the ileum in man. Am J Surg 121: 96-99.

7. Wangensteen $\mathrm{OH}(1978)$ Understanding the bowel obstruction problem. Am J Surg 135: 131-149.

8. Rana SV, Bhardwaj SB (2008) Small intestinal bacterial overgrowth. Scand J Gastroenterol 43: 1030-1037.

9. Cappell MS, Batke M (2008) Mechanical Obstruction of the Small Bowel and Colon. Med Clin North Am 92: 575-597.

10. Russel NS, Bulstrode JK (2008) Clinical features of intestinal obstruction, intestinal obstruction, bailey \& love's short practice of surgery (25th edn). Hodder Arnold, England. pp: 1188-1203.

11. Morris JB (2007) Etiology of small bowel obstruction, small bowel obstruction, shackelford's surgery, alimentary tract (6th edn). Saunders Elsevier, Philadelphia. pp: 1025-1035.

12. Ellis $H$ (1991) The cause and prevention of postoperative intraperitoneal adhesions; collective review. Surg Gynecol Obset 13: 497-511.

13. Stringer, Pablot SM, Brereton RJ (1992) Paediatric intussusception. Br J Surg 79: 867-876.
14. Cappell MS, Batke M (2008) Mechanical Obstruction of the Small Bowel and Colon. Med Clin J Am 92: 575-597.

15. Maglinte DD, Heitkamp DE, Howard TJ, Kelvin FM, Lap-pas JC (2003) Current concepts in imaging of small bowel obstruction. Radiol Clin North Am 41: 263 283.

16. Lappas JC, Reyes BL, Maglinte DD (2001) Abdominal radiography findings in small-bowel obstruction: relevance to triage for additional diagnostic imaging AJR Am J Roentgenol 176: 167-174.

17. Tavakkolizadeh A, Whang EE, Ashley SW, Zinner MJ (2010) Small bowe obstruction, small intestine, schwartz's principles of surgery (9th edn). Mc Grow Hill, USA. pp: 980-1012.

18. Hayanga AJ, Bass-Wilkins K, Bulkley GB (2005) Current management of smallbowel obstruction. Adv Surg 39: 1-33

19. Choi HK, Chu KW, Law WL (2002) Therapeutic value of gastrografin in adhesive small bowel obstruction after unsuccessful conservative treatment: a prospective randomized trial. Ann Surg 236: 1-6.

20. Lim JH, Ko YT, Lee DH, Lee HW, Lim JW (1994) Determining the site and causes of colonic obstruction with sonography. AJR Am J Roentgenol 163 1113-1117.

21. Suri S, Gupta S, Sudhakar PJ, Venkataramu NK, Sood B, et al. (1999) Comparative evaluation of plain films, ultrasound and CT in the diagnosis of intestinal obstruction. Acta Radiol 40: 422-428.

22. Furukawa A, Yamasaki M, Furuichi K, Yokoyama K, Nagata T, et al. (2001) Helical CT in the diagnosis of small bowel obstruction. Radiographics 21: 341 355

23. Al-Musawi D, Tompson JN (2001) Intra-abdominal Adhesions Formation and management. Recent advances in surgery (24th edn). Taylor I and Johnson, USA. pp: $1-19$

24. JJY Liauw, Cheah WK (2005) Laparoscopic Management of Acute Small Bowe Obstruction Asian J Surg 28: 185-188.

25. Bastug DF, Trammell SW, Boland JP (1991) Laparoscopic adhesiolysis for small bowel obstruction. Surg Laparosc Endosc 1: 259-262.

26. Kavic SM (2002) Adhesion and Adhesiolysis the role of Laparoscopy. JSLS 6 : 99-109.

27. Duepree HJ, Senagore AJ, Delany CP, Fazio VW (2003) Does means of access affect the incidence of small bowel obstruction and ventral hernia after bowe resection? Laparoscopy versus laparotomy. J Am Coll Surg 197: 177-181.

28. Wullstein C, Gross E (2003) Laparoscopic compared with conventional treatment of acute adhesive small bowel obstruction. Br J Surg 90: 1147-1151.

29. Tjandra JJ (2006) Operative treatment, small intestinal obstruction, text book of surgery (3rd edn). Blackwell Publishing, USA. pp: 159-164.

30. Russel NS, Bulstrode JK (2008) Clinical features of intestinal obstruction intestinal obstruction, bailey \& love's short practice of surgery (25th edn). Hodder Arnold, England. pp: 1188-1203.

31. Chosidow $D$, Johanet $H$, Montariol $T$, Kielt $R$, Manceau $C$, et al (2000) Laparoscopy for acute small-bowel obstruction secondary to adhesions. J Laparoendosc Adv Surg Tech A 10: 155-159.

32. Essani R, Bergamaschi R (2008) Laparoscopic management of adhesive small bowel Obstruction. Tech Coloproctol 12: 283-287.

33. Zerey M, Sechrist CW, Kercher KW, Sing RF, Matthews BD (2007) Laparoscopic management of adhesive small bowel obstruction. Am Surg 73: 773-778.

34. Chopra R, McVay C, Phillips E, Khalili TM (2003) Laparoscopic lysis of adhesions. Am Surg 69: 966-968.

35. Strickland P, Lourie DJ, Suddleson EA, Blitz JB, Stainls SC (1999) Is laparoscopy safe and effective for treatment of acute small-bowel obstruction? Surg Endosc 13: 695-698.

36. Farinella E, Roberto C, Francesco LM, Lorenzo C (2009) Feasibility of laparoscopy for small bowel obstruction. World Journal of Emergency Surgery $4 \cdot 3$

37. Lujan HJ, Oren A, Plasencia G, Canelon G, Gomez E, et al. (2006) Laparoscopic Management as the Initial Treatment of Acute Small Bowel Obstruction. JSLS 10: $466-472$. 
38. Franklin ME Jr, Gonzalez JJ Jr, Miter DB, Glass JL, Paulson D (2004) Laparoscopic diagnosis and treatment of intestinal obstruction. Surg Endosc 18: $26-30$

39. O'Connor DB, Winter DC (2012) The role of laparoscopy in the management of acute small-bowel obstruction: a review of over 2,000 cases. Surg Endosc 26: 12-17.
40. Ghosheh B, Salameh JR (2007) Laparoscopic approach to acute small bowe obstruction: Review of 1061 cases .Surg Endosc 21: 1945-1949.

41. Maglinte DD, Kelvin FM, Sandrasegaran K, Nakeeb A, Romano S, et al. (2005) Radiology of small bowel obstruction: contemporary approach and controversies. Abdom Imaging 30: 160-178.

42. http://library.med.utah.edu/WebPath/GIHTML/GI030.html 